\title{
JAK Inhibitor INCB047986
}

\author{
National Cancer Institute
}

\section{Source}

National Cancer Institute. JAK Inhibitor INCB047986. NCI Thesaurus. Code C111761.

An orally bioavailable inhibitor of Janus-associated kinases (JAK), with potential antineoplastic activity. Upon oral administration, INCB047986 specifically binds to and inhibits the phosphorylation of JAK, which affects JAK-dependent signaling and may lead to an inhibition of cellular proliferation in JAK-overexpressing tumor cells. The JAK-STAT (signal transducer and activator of transcription) pathway plays a key role in the signaling of many cytokines and growth factors and is involved in cellular proliferation, growth, hematopoiesis, and the immune response; JAK kinases may be upregulated in inflammatory diseases, myeloproliferative disorders, and various malignancies. 